\title{
Effectiveness of Teaching Program on Critical Care Nurses Performance Regarding Emergency Cart in Intensive Care Units
}

\author{
Sahar Gamal Khaliefa ${ }^{1}$, Olfat Abd Al-Ghany El-Shafiey ${ }^{2}$ \& Asmaa Aly Mahgoub Taher ${ }^{3}$. \\ Nursing Specialist, Abnoub Central Hospital, Assuit Governorate, Egypt. \\ Assistant Professor of Critical Care and Emergency Nursing Faculty of Nursing, Assuit University, Egypt. \\ Lecture of Critical care and emergency Nursing-Faculty of Nursing, Assuit University, Egypt.
}

\begin{abstract}
The emergency cart features is storing lifesaving supplies that are easy to transport and easily accessible in all parts of the cart for rapid seeing and removal of supplies and medications through a crucial situation. Aim: Evaluate the effectiveness of teaching program on critical care nurse's performance regarding emergency cart in intensive care units. Design: Quasi-experimental design. Setting: Emergency care unit, cardiology and neonatal intensive care unit at Abnoub Central Hospital Subjects: The target sample included 40 nurses (15) nurses from emergency care unit, (10) nurses from cardiology and (15) nurses from neonatal intensive care unit. Methods: Two information gathering tools were utilized. (Knowledge Assessment Tool) and (observation checklist).The present study was directed on evaluation of the level of nurse's information and abilities by using the two tools Then application where whole groups were passing four meetings and evaluation performed. Results: There were statistically significant differences between intervention before and after regarding nurse's knowledge (57.5\%) and practices $(82.5 \%)$. Conclusions Educational program was useful on improvement of nurse's performance regarding emergency cart. Recommendations: All nurses must be aware with all equipment stored on the emergency cart.
\end{abstract}

\section{Key words: Emergency Cart, Intensive Care Unit, Teaching program \& Performance of Critical Care} Nurses.

\section{Introduction}

The success or failure of cardiopulmonary resuscitation (CPR) implementation in a hospital depends on the efficiency of the nurse or doctor and on the sufficiency of the emergency cart's component. In order to perform successful CPR, the life-saving supplies of the emergency cart must be standardized. If the contents of preceding emergency cart have been checked for deficiencies, then addressing these deficiencies can save patients' lives. (Rajeswaran \& Ehlers, 2012 ).

The emergency cart is a portable trolley containing all equipment and drugs for cardiopulmonary resuscitation and emergency need. Crash cart maintenance is a method of keeping unit based crash cart properly supplied, organized and maintained. (Ministry of health, 2013).

The purpose of Crash Cart is to provide supplies and equipment used by a team of provider to provide emergent care when the patient is experiencing a life - threatening situation/ Crash carts are designed to contain specific supplies and medications, They must be maintained in areas where they are implemented patient care activities and must be ready for use all times. (Welker et al., 2016).

The function of crash carts is to provide a moveable station within the hospital that contains everything needed to treat a life threatening situation. The advantage of mobility is that it allows the treatment to come to the patient when needed (Wheeler, 2012) The contents of an emergency cart differ from hospital into hospital; however commonly include the tools and drugs required to treat someone in or nearness cardiac arrest. These contain however, are not bounded to: Monitor, defibrillators, suction devices, Advanced cardiac life support (ACLS) drugs such as adrenaline, atropine, lidocaine, sodium bicarbonate, dopamine, and vasopressin, ......etc , IV solution, IV equipment and emergency intubation equipment . Drugs and other equipment selected by the facility (Gladstone, 2010).

The arrangement of the trolley must be having basic protocol including the location and the monitoring protocols throughout the institution that should be performed on a regular schedule, ideally with storage of appropriate disposable and non- disposable medical supplies and medications. (Makkar \& Madaan 2016) There is a focus on basic protocol as it increases the familiarization of the staff using these emergency carts and decrease opportunities of errors and confusion during a crucial situation (Colquhoun et al., 2012)

Organized emergency trolleys provide structure, saves time and decreases confusion in an otherwise possible chaotic situation. Every effort should be made to be able to streamline restocking of used or missing items required on the emergency trolley

(D Maritz et al., 2010). 
Critical care nurses' role the primary role consists of crash cart awareness, equipment familiarity, skills in handling the equipment, defibrillator safety and accurate documentation. Nurses are also responsible for checking resuscitation trolleys on a daily basis and ensuing that the trolleys are readily available in all clinical areas. The nurses regularly confirm all resuscitation items are in good working condition, and the trolley is kept locked for emergency resuscitation care. (B Ouseph et al., 2015).

\section{Significance of the study}

The first responders in resuscitation should be adequately prepared to provide effective cardiopulmonary resuscitation, therefore this study can guide the hospital personnel to keep standardized equipment in the resuscitation trolley in order to avoid confusion of their use.

\section{Aim of the study}

Evaluate the effectiveness of teaching program on critical care nurse's performance regarding emergency cart in intensive care units

Hypothesis

- Significant difference in the nurse's performance before and after the test.

- Nurse's performance enhanced after the teaching program.

\section{Subjects \& Methods}

Research Design: Quasi-experimental design (pre \& post) testing was used for Evaluation the effectiveness of the structured teaching program of the current study.

Variables: Independent variable was teaching program. While the dependent variable was the nurse's performance in critical care units .

Settings: The study was done at Abnoub central Hospital in the emergency care unit, the coronary care unit and the neonatal intensive care unit.

Subjects: The target sample (The total number was 40 nurses) were included in the study from the above mentioned study settings.

Inclusion criteria Nurses were included in this study were from both gender, with different age, educational levels and years of experience.

Exclusion criteria: Nurses who wasn't want to participate in the study.

Study tool: Two tools were used in this study.

Tool I (pre-post emergency cart knowledge questionnaire for nurses): This tool consisted of two parts

Part I: Demographic data for nurses including: demographic characteristics of studied nurses such as age, gender, qualification, long periods of experience and attendance of relevant training courses. (Assessment of personal data of the nurse)

Part II: Emergency cart knowledge questionnaire (included 15 Structured multiple choice questions), developed by the researcher based on the relevant literature (Welker et al., 2016 \& Jacqueline Wheeler, 2012) to assess Nurses 'knowledge regarding emergency cart and it consists of Knowledge regarding emergency cart as definition of emergency cart, purpose, contents, function of the crash cart, drugs arrangement and equipment in the emergency cart and role of critical care nurses.

The scoring system: The total score of knowledge was 30 degree. The score two was given for each entire right answer, score one was given for each inadequate right answer, and score zero for the wrong answer. For each area of knowledge, the scores of the items were summed up and the total score divided by the number of the items. Next the grades were converted into a percent score. The total nurses' information was considered satisfactory if the percentage was at least $60 \%$ or more, and unsatisfactory if under $60 \%$.

Tool II: Pre-post emergency cart observation checklist. (Emergency Crash Cart Checklist, 2010 \& Ministry of health, 2013): A checklist for observation has been created and developed by the researcher based on the relevant literature and validated by the five experts in critical care nursing department to assess nurses' practice regarding the following crash cart parameters Contents, labeling, equipment functions and documentation.

The scoring system: The overall score of practice was 49 degree. The things observed to be done effectively were scored "1" and the things that were not done, or ineffectively done were scored"0". For each procedure, the grades of the total things were summed - up and next divided by the number of the items. These grades were changed over into a percent score. This practice was considered satisfactory if the percentage was at least $60 \%$ or more, and unsatisfactory if under $60 \%$.

\section{Teaching Program}

Based on the previous assessment of nurse's information and practices, accessible resources and review of literature the researcher designed the teaching Program

General objectives of the teaching program:

The overall objectives of the designing teaching program was to increase the knowledge and practices of the nurses about emergency cart in critical areas.

The specific objective of the program:

By the end of the program nurses were able to:

1) Knowledge and understanding practices:

- Define the emergency cart.

- Identify the purpose of emergency cart. 
- Identify the emergency cart's function.

- Recognize the emergency cart's contents.

- List the arrangement of medication and equipment in the crash cart.

- Recognize the critical care nurses 'role regarding emergency cart.

\section{2) Professional skills}

- Checking the function of the equipment.

- Checking the expiration date of drugs and equipment

- Checking the emergency cart labeling.

- Perform in the emergency cart checklist.

3) Intellectual Skills

- Compare between types of antidysrthymia drugs.

- Summarize side effect for each drug.

General and transferable abilities

- Communicate effectively with the team of work.

\section{Methods}

Four consecutive stages: assessment, preparation, implementation and evaluation were used to conduct the study. Collection of data was done before and after implementation from February 2017 to July 2017.

Assessment phase: This phase aimed to assess the nurse's knowledge and practice in relation to Emergency crash cart.

\section{Preparatory phase}

- From the manager of Abnoub Hospital and furthermore, the head of the departments in which the study was conducted an formal order was achieved to gather the essential information after clarification the aim of the study and the thought of this study.

- Dependent upon inspection the relevant literature, the researcher building the tool used in this study was in Arabic language.

- By group of five specialists in critical care nursing, the tool was examined to validate its contents.

- Reliability was examined for tool one and tool two by using Cronbach's coaefficient alpha(r=0.827, 0.859 respectively) which is acceptable.

- Then, a pilot study was carried out in front of beginning collection of data on 4 nurses for testing the tool's feasibility and applicability, Then the necessary modification was done.

- After explaining the purpose and idea of the study, permission was obtained for voluntary participation of nurses.

- From the local ethics committee, an confirmation was achieved and the study was followed the usual ethical principles in clinical research.

- According to the assessment phase, program's content and media as the program booklet and visual materials were prepared by the researcher. According to the opinion of a panel of expertise some modifications were done and followed by were developing the final forms.

Development of the teaching program: The program was developed by the researcher, after reviewing the relevant literature. The next steps have been adopted to develop the program.

Program planning: the program's content was arranged into four sessions in addition to introductory one. The program's content included two parts related to:

- Information about emergency cart and emergency medications.

- Practice of procedure required for organizing, arrangment and restocking of emergency cart.

The teaching methods utilized were real situations, modified lectures, demonstration and group discussions,.

An instructional media were included; a program booklet and audiovisual materials were utilized. The majority of the studied nurses were cooperative with the researchers in all places of study. The studied nurses were interested in the topic and they asked to repeat such this program for nurses in different health care settings

Implementation phase: It included two parts: -

1- Theoretical part: Designed by the researcher based on the related literature and consists of definition of emergency cart, purpose, contents, function of the crash cart, drug arrangement and equipment in the crash cart and critical care nurses 'role.

2- Practical part: the supervision checklists were completed by the researcher who was available five days a week. By the studied nurses the questionnaire was filled in the clinical field in the presence of the researcher. All nurses were 40, divided into eight main groups according to study setting and next the program was applied in the pre-mentioned study settings for each group singly according to their requirements. The period of each session was about 15-20 minutes. The sessions were beginning according to nurse's leisure time, and the Arabic language was utilized to be suitable for the level of nurses' understanding.

3- Evaluation phase: The evaluation phase was stressed on assessing the effect of the teaching program on nurses 'knowledge and practice in relation to emergency crash cart and its 'organization, The same pre- test study tool (questionnaire and observational checklist) was used as post- test for studied subjects . 


\section{Statistical design}

By using the statistical package for social science (SPSS), version (11), the collected data were arranged, encoded, computerized, tabulated and analyzed. Data analysis was completed by the utilization of number, percentage distribution; mean, standard deviation, and Independent sample $\mathrm{T}$ test, chi-square test-one way ANOVA, analyses was used to test the significance of some variances. A significant level value was considered when $\mathrm{p}<0.05$, non-significant (NS) if $P>0.05$, Highly Significant (HS) if $P<0.01$.

\section{Results}

Table (1): Distribution Demographic data for the studied nurses $(n=40)$.

\begin{tabular}{|c|c|c|}
\hline \multirow{2}{*}{ Nurses socio-demographic data } & \multicolumn{2}{|c|}{ Group } \\
\hline & No & $\%$ \\
\hline \multicolumn{3}{|l|}{ Gender } \\
\hline Male & 6 & 15.0 \\
\hline Female & 34 & 85.0 \\
\hline \multicolumn{3}{|l|}{ Age } \\
\hline 20 to $<30$ & 39 & 97.5 \\
\hline 30 to $<40$ & 1 & 2.5 \\
\hline Mean \pm SD & \multicolumn{2}{|c|}{$28.5 \pm 5.4$} \\
\hline \multicolumn{3}{|l|}{ Nursing qualification } \\
\hline • Diplom nursing. & 21 & 52.5 \\
\hline - Technical institute. & 18 & 45.0 \\
\hline - Bachelor of nursing. & 1 & 2.5 \\
\hline \multicolumn{3}{|l|}{ Experience level } \\
\hline 1 to $<5$ years & 13 & 32.5 \\
\hline 5 to $<10$ years & 27 & 67.5 \\
\hline Mean \pm SD & \multicolumn{2}{|c|}{$7.1 \pm 4.0$} \\
\hline \multicolumn{3}{|l|}{ Training } \\
\hline Yes & 8 & 20.0 \\
\hline No & 32 & 80.0 \\
\hline \multicolumn{3}{|l|}{ Target ICU\& Units } \\
\hline Emergency unit & 15 & 37.5 \\
\hline $\mathrm{CCU}$ & 10 & 25.0 \\
\hline NICU & 15 & 37.5 \\
\hline
\end{tabular}

Table (2): Relation between nurses' practice (Pre \& Post - test) related to departments (Emergency unit, ICU and NICU).

\begin{tabular}{|c|c|c|c|}
\hline & Pre & Post & \multirow{2}{*}{ P-value } \\
\hline & Mean \pm SD & Mean \pm SD & \\
\hline - Organized and crash cart system & $3.6 \pm 0.5$ & $18.6 \pm 0.5$ & $0.0002 * * *$ \\
\hline - Crash cart daily checklist & $8.0 \pm 2.0$ & $17.0 \pm 1.7$ & $0.004 * *$ \\
\hline \multicolumn{4}{|l|}{ - $\quad$ Adult crash cart nursing checklist } \\
\hline $1^{\text {st }}$ Drawer of crash cart(emergency medications) & $9.6 \pm 2.5$ & $12.0 \pm 2.0$ & 0.277 \\
\hline $\begin{array}{l}2^{\text {nd }} \text { Drawer of crash cart (Airway Management } \\
\text { Equipment) }\end{array}$ & $10.0 \pm 0.0$ & $18.6 \pm 2.3$ & $0.003 * *$ \\
\hline $3^{\text {rd }}$ Drawer of crash cart (IV Essential Equipment) & $5.6 \pm 2.0$ & $10.6 \pm 2.3$ & 0.050 \\
\hline $\begin{array}{l}4^{\text {th }} \text { Drawer of crash cart ( Dextrose } 5 \% 500 \mathrm{ml} \text {, Ringer } \\
\text { Lactate } 500 \mathrm{ml} . . . \text { etc) }\end{array}$ & $3.6 \pm 4.0$ & $4.6 \pm 4.1$ & 0.780 \\
\hline $\begin{array}{l}5^{\text {th }} \text { Drawer of crash cart (Sterile towels, A calculator } \\
\text { and stethoscope, Penlight). }\end{array}$ & $8.0 \pm 6.6$ & $6.6 \pm 4.1$ & 0.791 \\
\hline
\end{tabular}




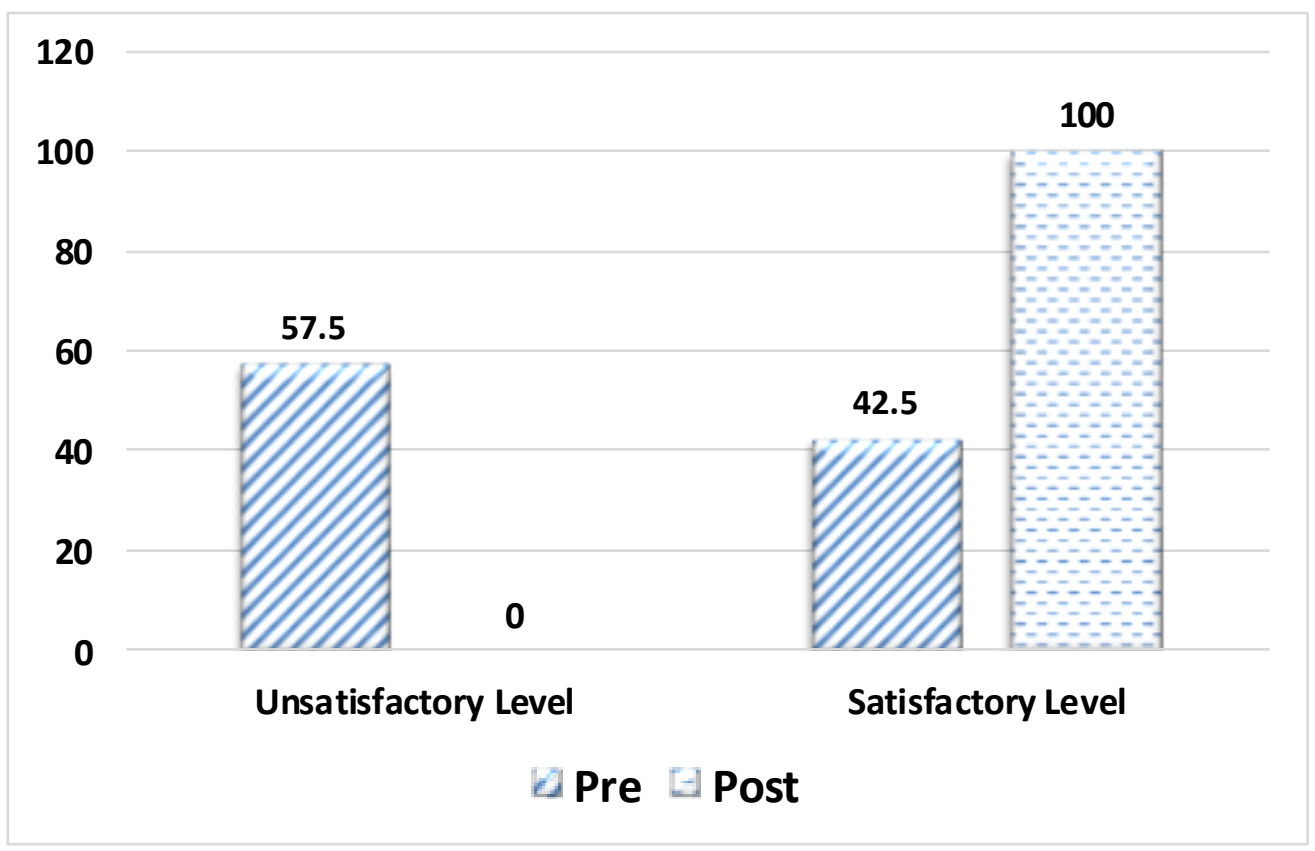

Figure (1): Percentage distribution of nurses' knowledge

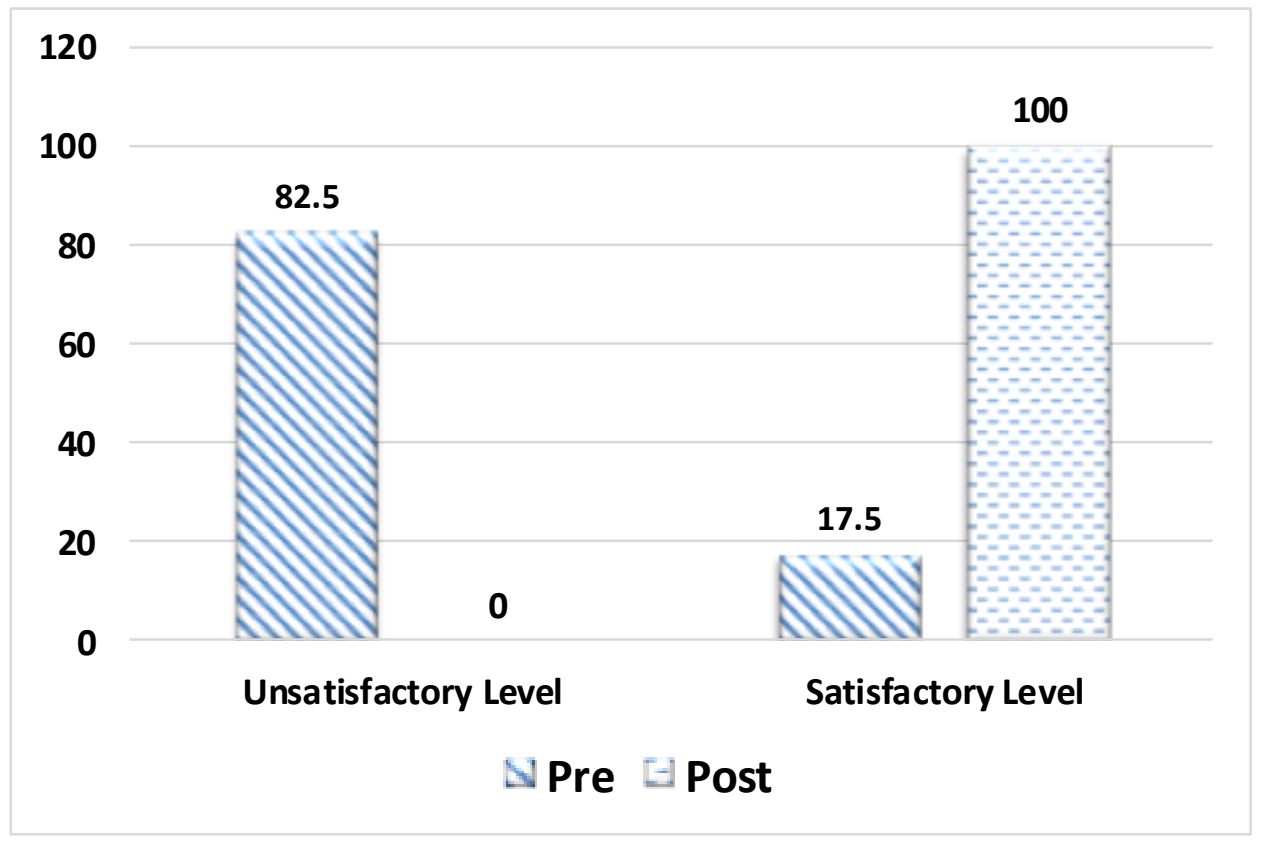

Figure (2): Percentage distribution of nurses' practice

Table (1): Show that Most of studied nurses were female $(85.0 \%)$, hade aged between 20 to $<30$ years $(97.5 \%)$ and about half of them $(52.5 \%)$ were diplom nursing. regarding years of experience more than two third of studied nurses $(67.5 \%)$ had 5 to <
10 years of experience. Most of studied nurses $(80.0 \%)$ hadn't received any prior training.

Table (2): Show that There is very highly statistical significant differences between nurses' practice (pre \&post-test) related to nurses' departments at (Organized and unorganized crash cart system) with 
$\mathrm{p}$-value at (0.000), and also there are highly statistical difference between nurses' practice (pre\& post -test) related to nurses' department at (Crash cart daily checklist and drawer(Airway management equipment) with $\mathrm{p}-$ value $(0.004$ and 0.003 respectively)

Figure (1): show that There is a statistical significant difference between nurses' knowledge among study nurses pre and post. The most of nurses had unsatisfactory level pre-knowledge with percentage $(57.5 \%)$. All satisfactory levels of nurses' knowledge were in Post Study group with percentage (100.0\%).

Figure (2): Show that There is highly statistical difference regarding nurses' practice between study nurses pre and post. The larger part of nurses had unsatisfactory level pre-practice with rate $(82.5 \%)$. All satisfactory levels of nurses' knowledge were in Post-test with percentage (100.0\%).

\section{Discussion}

The successful management of cardiopulmonary crisis cases rotates around the perfect utilization of the golden hour, in order that the patient has the better possible survival advantage. To accomplish this, and to enhance response time in cardiopulmonary resuscitation (CPR) management, emergency carts/ resuscitation trolleys are utilized for which a strict arrangement of the teams and equipment and supplies is required (Makkar\& Madaan, 2016)

The present study demonstrate that vast majority of the nurses who varied in their level of education, half of them had diploma degree and nearly half of the nurses had Technical institute degree. The greater part of nurses had unsatisfactory level pre-knowledge and all satisfactory levels of nurses' knowledge were in post- test.

This could be attributed to the fact that nurses in Egypt don't utilize the independent self-learning. Another cause for lack of knowledge nurses' exhaustion in consequence of raised work load which may hinder their capacity to read and bring up to date their information. This might be related to the fact that vast majority of them have nursing diploma and majority of books are written in English and their learning in Arabic This result is in concurrence with Raja et al., (2009) who was conducted a descriptive study aimed to assess the knowledge, skills and competency regarding medical emergency crash cart and use of emergency medications .the study finding revealed that there is superficial knowledge on medical emergencies, drugs and equipment among health professionals working in emergency unit, but after the education and practical demo the knowledge of the nurses was increased.
Also, this result is in agreement with (Paramesha\& kumar, et al., 2016) who Assess the Effect of Self Instructive Module of Knowledge on using of Emergency Crash Cart System in Hospital between 4th year B. Sc Nursing Students of chosen Nursing Colleges in Mysore findings of Pretest revealed that 4th year B. Sc Nursing students were having low level of knowledge. After giving Self-instructional module post test score increased, which shows the effect of Self-instructional module.

Regarding the result of the nurses' knowledge scores concerning Emergency Cart the present study provided with documentary evidence significant increase in application of the post-knowledge program for nurses This might be connected to the fact that this knowledge is insufficient with regard to the emergency Cart Additional cause is the absence of before-appointment direction and in-service teaching programs concerning Emergency Cart. These findings were consistent with Khemnar et al., (2017) found that the structured teaching program was found to be significantly effective in improving the knowledge of the fourth years B.Sc. nursing students regarding utilization of crash cart. This indicates that there is remarkable improvement in the knowledge of the 4th year B.Sc. nursing students regarding utilization of crash cart. Also, this result is in agreement with Rajendra \& Lamkhede (2014) who evaluated the Effect of the planned teaching program on knowledge about emergency drugs between nurses that the knowledge of nurses after pre-test and before planned teaching program was not satisfactory; the planned teaching program helped them to learn about emergency drugs. The score of knowledge after the test exhibited significant increase in knowledge.

With respect to the performance of nurses, the findings of the present study supported hypothesis (II), i The finding of the present study exhibited that the Majority of nurses had a pre-practice level that was unsatisfactory and All satisfactory levels of nurses' practice were in Post Study This may be connected to the fact that majority of them are youth, secondary school nurse, not receiving any prior training. These results were In a similar line with Hamdi (2015) \& Marzooq\& Lyneham (2009) they found that the majority of nurses had unacceptable practice regarding crash cart (content \& preparation) and greater part of nurses had unacceptable performance level about emergency drugs.

The findings of the present study exhibited that there were highly statistically significant differences regard to the mean score of total practice in both pre and post teaching program including daily crash cart checklist and organized and unorganized crash cart system). This improvement in nurses' practice might 
be caused by the fact that the educational program stressed on the Practical training to change nurses' practice, which is needed for achievement of the desired level of practice. This goes in a similar line with Chacko (2015) who was revealed that majority of the nurses had (70\%) had average knowledge, $(23.3 \%)$ had weak knowledge and $(6.7 \%)$ had good knowledge regarding organized emergency crash cart and medications utilized in the hospital.

\section{Conclusions}

The teaching program was helpful on improvement of nurse's performance regarding emergency cart.

\section{Recommendations}

Dependent upon the finding of the present study, recommendations that are suggested are the following:

- Continuous training programs to update the knowledge and skills of nurses working in emergency units.

- An intermittent assessment of critical care nurses knowledge and skills concerning different nursing procedures at the intensive care units.

- To generalize the results, the study might be repeated on large sample and different hospitals setting.

\section{References}

1. Chacko (2015): A descriptive Study To Assess The Knowledge OF Organized Emergency Crash Cart And Medications Among Student Nurses Working In Selected Hospitals At Belagavi, KLE university, Belagavi, Karnataka, REG NO:MCO113001 .

2. Colquhoun M., \& Gabbot D., \& Mitchell S., (2012): Cardiopulmonary resuscitation guidance for clinical practice and training in primary care', Resuscitation Council (UK) viewed 21 February 2012, available at http://www.resus.org.uk

3. Emergency Crash Cart Checklist (2010): http://www.patient Safety authority.org /ADVISORIES/advisory library/2010/ jun 7 (2)/page52.asp

4. Gladstone J., (2010): Drug administration Errors, A study into the factors underlying the occurance and reporting of drug errors in a district General hospital Journal of advanced Nursing,(22),628637

5. Hamdi N., (2015): Assessment Of Nurses Performance During Cardiopulmonary Resuscitation In Intensive Care Unit And Cardiac Care Unit At The Alexandria Main University Hospital, International Journal Of Scientific \&
Technology Research .Vol.(4)No(12), 141-148, www.ijstr.org

6. Jacqueline Wheeler. Thinking your way to successful problem- solving [homepage on the internet](2006): [cited 2012 march 6]. Available from: http://www.nursingtimes.net/nursingpractice-clinical- research/thinking- your- way-tosuccessful-problem-solving/ 200636.article

7. Khemnar A., \& Karkar M., \& Nagin Y., (2017): An experimental Study To Assess The Effectiveness Of structured Teaching Programm On Knowledge Regarding Utilization Of Crash Cart In Hospitals Among 4th year b.sc nursing Students Of Selected Nursing Colleges In Pune City, International Journal of Applied Research 2017.vol (3) No(7), 469-473.

8. Makkar N., \& Madaan N., (2016): Study of compliance of crash carts to standards in the emergency of a tertiary care teaching hospital, International Journal of Research in Medical Sciences September .Vol .(4) NO (9) .

9. Maritz D., \& Hodkinson P., \& Wallis L., (2010): Identification of performance indicators for emergency centres for South Africa: Results of a Delphi Study. International Journal of Emergency Medicine 3:341-349

10. Marzooq \& Lyneham, J., (2009): Cardiopulmonary resuscitation knowledge among nurses working in Bahrain', International Journal of Nursing Practice 15(4) 294-302 http://dx.doi. org/ 10.1111/j.1440- 172X.2009.01752.x

11. Ministry of Health (2013): king Fahd National Library Cataloging in-publication Data, Guidelines For Emergency Department, General Directors of health centers-Riyadh,ISBN:978603-8144-052, www. mohp. gov.eg.

12. Ouseph B., \& Mohidin S., Tabsh L., \& AlHebsh A., (2015): Nurses' Resuscitation Performance: Study on the Effectiveness of Training and Support at a Teaching Hospital in KSA, International Journal of Cardiovascular and Cerebrovascular Disease vol. (3) No (4): 21-27.

13. Paramesha, Vinay Kumar, G., vishakanta Murthy, D., (2016): A study to Assess the Effectiveness of Self Instructional Module of Knowledge on Utilization of Emergency Crash Cart System in Hospital among 4th year B. Sc Nursing Students of Selected Nursing Colleges in Mysore . Asian J. Nur. Edu. and Research. 2016; 6(2): 209-213.

14. Raja Fazline D., \& Syed Z., (2009): Knowledge ,attitude , and practice of nurses in administering oral medication at medical ward .University Kebangsaan Malaysia Medical Center Med \&Health.4 (1):16-24 
15. Rajendra D., \& Lamkhede (2014): Effectiveness of planned teaching programme on knowledge of emergency drugs among staff nurses ,Sinhgad e Journal of Nursing, Vol. IV http://www. sinhgad.edu/ sinhgadnursingcollegejournal/Vol_IV_Issue_I/12. pd

16. Rajeswaran L., \& Ehlers V., (2012): Audits Of Emergency Trolley's Contents In selected hospital In Bostswaran .vol.(17)No(1),1-7.

17. Welker R., Mueller T., (2016): CHAN health care, Clinical minute, Crash Cart Readiness , available at https:www. Crowehorwat . com/pdf/ clinical minute

18. World Health Organization (WHO) (2009): available at http:// www. who. int/ countries/ egy/en/ 